\title{
Generic Service Location Discovery Recommender System for Mobile Users
}

\author{
Tranos Zuva and Keneilwe Zuva
}

\begin{abstract}
Recommender systems are information filtering and decision support tools aimed at addressing problems encountered by online browsers. Recommender systems have been applied in many diverse areas including e-commerce, advertising, news, document management and e-learning. The existence of mobile technologies that offer online services anywhere and anytime would make recommender systems useful to mobile users. This research designed and developed a service location discovery recommender system that can help mobile users to find places where they can receive services best suited to them. Experimental results were generated and analyzed to test the efficacy of the generic service location discovery recommender system for mobile users.
\end{abstract}

Index Terms - Recommender system, decision support, information filtering, evaluation metrics.

\section{INTRODUCTION}

Recommender systems belong to a class of personalized information filtering technologies that aim to meaningfully suggest which items or products available might be of interest to a particular user [1], [2]. In most cases these systems overcome the problem of information overwhelming or lack of specific knowledge by providing users with personalized items, services, service location or products recommendations. The existence of mobile technologies that offer online services anywhere and anytime would make recommender systems useful to mobile users. The service location discovery recommender systems can help mobile users to find places where they can receive services best suited to them. The recommender systems make recommendations using three fundamental steps: preferences acquisition (acquiring preferences from the user's input data), recommendation computation (computing recommendations using proper methods) and recommendation presentation (presenting the recommendation to the user) [3], [4].

Based on various techniques used in recommendation computation existing recommendation systems can be classified into four fundamental categories, that is, Collaborative Filtering (CF), Content-Based Filtering (CBF), Knowledge-Based filtering (KBF) and Hybrid Filtering (HF) [5].

The goal of the service location discovery is to efficiently find a set of services that best match user desired service. The goal of this study is to:

- Minimize the location proximity between a user and a recommendation

Manuscript received March 25, 2014; revised July 23, 2014

The authors are with the Department of Computer Systems Engineering, Soshanguve South Campus, South Africa (e-mail: tzuva@hotmail.com, kzuva@yahoo.ca).
- Maximize the similarity between a recommendation and a query

- Minimize the gap between the service requirements and what the user can offer.

The remainder of this paper is organized as follows: Section II the proposed recommender system, Section III the recommendation method employed, Section IV the results of recommendation system and Section $\mathrm{V}$ conclusion and future work.

\section{PRoposed RECOMMENDER SySTEM}

The proposed recommender system is supposed to use the Content-Based Filtering (CBF) applying the multi-criteria ratings. This provides more information about user preferences regarding various aspects of the service. The significance of multi-criteria recommender systems is to find personalized services for users anywhere anytime. The system developed is Client-Server architecture. The client consists of the mobile application that captures the user profile and tastes at the initial stage. The client also has the GPS position of the client at any given time when the application is in use. The Server consists of the application that is used to capture the service record and to produce the service profile of the service locations the client visited. It has the recommendation generator that generates the recommended service locations to the user. The proposed recommender system is shown in Fig. 1.

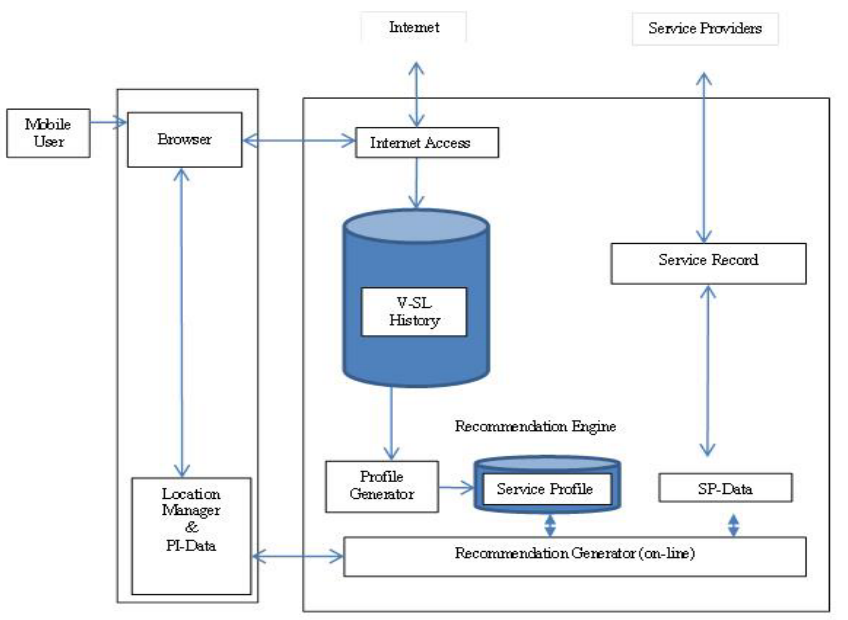

Fig. 1. Recommender system architecture.

\section{RECOMMENDATION METHOD}

$\mathrm{CBF}$ approaches recommend items that are similar in content to the items the user liked in the past or march to the attributes of the user [6], [7]. In this study content based filtering recommender system recommends service locations 
that are similar in content to the attributes of the user or to the service locations that the user liked in the past. Every service location is represented by a feature vector or an attribute profile. The feature hold numeric or nominal values representing certain aspects of the service location like GPS coordinates, mode of transportation, mode of payment, etc. The similarity measure between the feature vectors was used to compute the similarity of two service locations or between the user profile and service location. The Euclidean dissimilarity algorithm was used and is given in (1).

Euclidean dissimilarity

$$
\left.\operatorname{dissim}(x, y)=\sqrt{\sum_{i=1}^{n}\left(x_{i}\right.}-y_{i}\right)^{2}=\|x-y\|_{2}
$$

where $x$ and $y$ are service location vectors with $n$ elements in them, $\operatorname{dissim}(x, y)$ measures the distance apart of the service locations being compared or between the desires of the mobile user and what the service location is offering.

The similarity value is then used to obtain a ranked list of recommended service location. This approach is based on information retrieval because content associated with the user's preferences is treated as a query and unrated objects are scored with similarity to the query.

\section{A. Service Location and User Profile Representation Modelling}

The main aim of the service location representation is to model attributes that are common to large classes of service locations. This was achieved by capturing important service location information such as service type, name, mode of payment (card (credit and/or debit), cash and/or free), accessibility (public and/or private), pricing (negotiable, fixed and/or discounted) and GPS location. Particular features unique to each service location are also captured into the database. The recommendation method represents service location information as a feature vector of $n$ values as shown in (2).

$$
s=\left(s_{1}, s_{2}, s_{3}, s_{4}, s_{m}, \ldots \ldots \ldots \ldots, s_{n}\right)
$$

where $S_{i}$ may be numeric, nominal or a set of number, $S_{1}$ represents service type, $s_{2}, \ldots, s_{m}$ represent the common attributes of all service locations and $s_{m+1}, \ldots \ldots, S_{n}$ represent the unique attributes in a service type.

The example of service location feature vector as shown in (3):

$$
\begin{array}{r}
s=(\text { Gas Station, cash, private, fixed, Day } \& \\
\text { Night }, \ldots \ldots,\{78.34,38.61\}, \text { air presure })
\end{array}
$$

The mobile user profile is modelled as feature vector $m$ values shown in (4).

$$
u=\left(u_{1}, u_{2}, u_{3}, u_{4}, \ldots \ldots \ldots \ldots \ldots \ldots \ldots \ldots \ldots, u_{m}\right)
$$

where $u_{i}$ may be numeric, nominal or a set of number, $u_{1}$ represents user ID, $u_{2}, \ldots ., u_{m}$ represent the common attributes of all the service locations rated by the user before initiating a query. Different scales can be used to service location attributes [4]. In this research the scale of five (5) stars was used with the following meaning to the stars: 1 starI hate it; 2 stars- I don't like it; 3 stars- Its ok; 4 stars- I like it; 5 stars- I love it.

The example of mobile user feature vector as shown in (5).

$$
\begin{aligned}
u= & (\text { UserID },(\text { cash }=5, \text { credit }=1, \text { debit }=3), \\
& (\text { private }=1, \text { public }=5), \\
& (\text { fixed }=5, \text { negotiable }=4, \text { auction }=3), \\
& (\text { Day }=5, \text { night }=1) \ldots \ldots . . . .)
\end{aligned}
$$

In the user feature vector the scale used

\section{B. Illustration of the Operations of the System}

Initially the mobile user rates the generic service attributes of service locations as shown in equation (5). The user requests for a service location of a certain service type, for example a gas station by clicking on the appropriate icon.

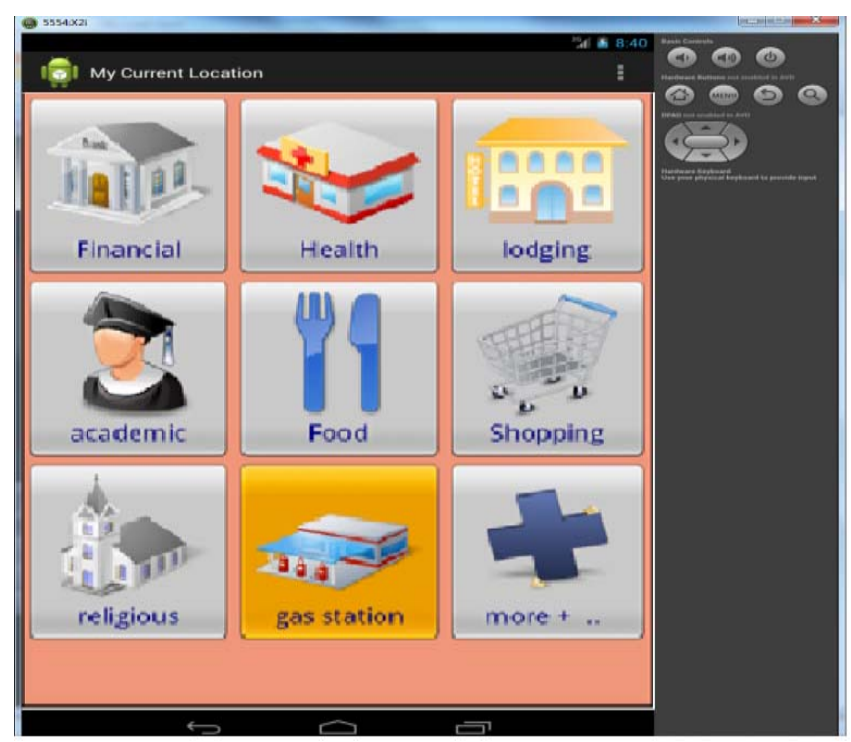

Fig. 2. Interface showing icons of service types.

The request is sent to the servicer together with GPS coordinates of the user. The system then finds a number of service locations for example ten (10) considered to be the nearest to the user location.

In this research the distance is calculated using the following formula [8]:

$$
\begin{aligned}
d_{u L}= & 2 \times \operatorname{asin}\left(\operatorname{sqrt}((\operatorname{lat} 1-\operatorname{lat} 2))^{2}+\cos (\operatorname{lat} 1)\right. \\
& \times \cos (\operatorname{lat} 2) \times(\sin ((\operatorname{lon} 1-\operatorname{lon} 2) / 2))^{2}
\end{aligned}
$$

where $\{$ lat $1, \operatorname{lon} 1\}$ and $\{$ lat $2, \operatorname{lon} 2\}$ are GPS coordinates for the mobile user and the service location respectively. It is very important to note that lat and lon stand for latitude and longitude respectively. North latitudes and west longitudes are taken as positive and south latitudes and east longitudes are taken as negative.

The system, from the nearest service locations found, it calculates the distance between the desires of the mobile user and the requirements of the service location. Equation (1) is used to calculate the dissimilarity. Taking the mobile user's desires as in (4) and the requirements of the service location 
as in (2), the calculation of the dissimilarity is shown in (7).

$$
\operatorname{dissim}\left(u, s_{1}\right)=\sqrt{\sum_{1}^{4}((5,5,5,5)-(5,1,5,5))^{2}}=4
$$

The system recommends the service locations with lowest dissimilarity, showing all their attributes (including the unique to that particular service location). The mobile user makes the final decision to which service location to go. The system then starts to build a database of service locations the mobile user desires. The database is created when the mobile user visits a service location, the system would find the location in the server database and the user confirms the service location as shown in Fig. 3.

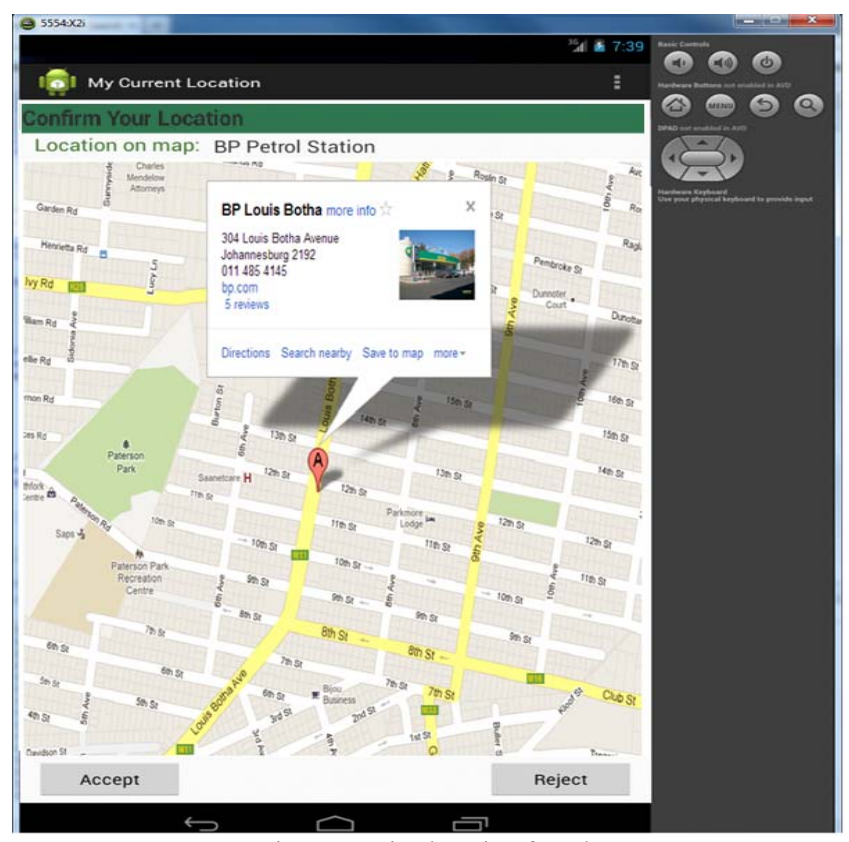

Fig. 3. Service location found.

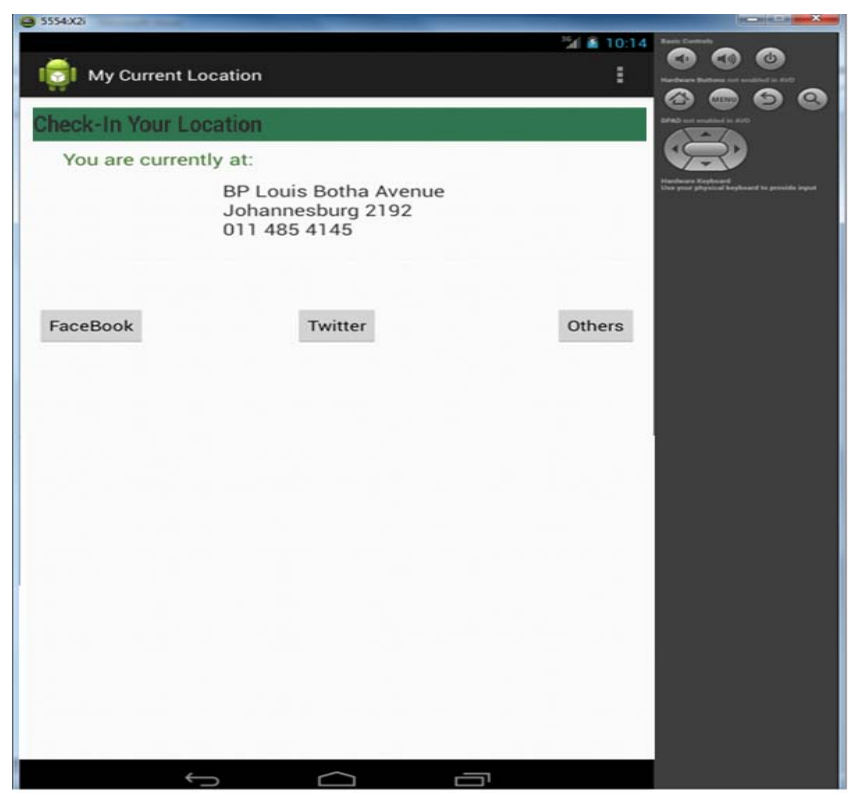

Fig. 4. User checks-in location.

When the service location has been confirmed the user checks-in the location to Facebook, twitter or others shown in Fig. 4. The system then records the service location in the database. When the database has reached a certain threshold the system then starts to compare the attributes of service location that the user liked before to recommend the next service locations to the user. The database is kept at a certain threshold of number of service location history, meaning when the threshold is reached the older service locations are dropped so that the system can adapt to changes of the mobile user. This enables the system not to explicitly request new ratings of the service locations from the mobile user.

\section{Evaluation OF RECOMMENDER SySTEM}

The most often and significant basic offline measures for recommender systems effectiveness are precision and recall [9]-[11]. Precision is the fraction of recommended items that are relevant to all recommended items in (8). Recall is the fraction of relevant items that are recommended to relevant items in the database given in (9) [9].

The formulas for Precision $(P)$ and Recall $(R)$ are below in (8) and (9).

$$
\begin{gathered}
P=\frac{A}{A+C} \\
R=\frac{A}{N}
\end{gathered}
$$

where $A$ is the number of relevant items recommended, $C$ is the number of not relevant items recommended and $N$ is the total relevant items in the database.

To the user the scalar value of recall indicates the ability of the system to find relevant items as per query from the collection of different items. Precision shows the ability to output relevant items as per query. In general the user is interested in the relevant recommended items. Thus the measures of precision and recall become measures of interest to the users. The lower the values indicate undesirable performance of the system.

The higher the values the more the user is encouraged to use the system due to the anticipation of getting more of the relevant search items. The Recall-Precision graph gives a visual performance of the system at different stages. The visual performance measure preserves all performance related information about a recommender system. The visual performance measure is capable of showing if one system dominates the other system totally or partially. These performance measures were used to measure the recommender system.

\section{EXPERIMENT AND RESULTS}

The experiment conducted to evaluate the recommender system was done offline. Selected services were experimented with and the users were selected to rate the common attributes of the services. The recommender system was then used to rank the service locations from one to five from ten service locations offering the service. The users were also requested to rank the service locations they would visit from one to five. In all the lists one would be the most preferred and ten the least. The user list was then used to evaluate the recommender system. The system was experimented with fifty (50) users and just ten (10) service 
types. The following results illustrate how the recall and precision was calculated. The following data was taken from one of the fifty (50) users for a given service.

The service locations were labelled as:

$$
A, B, C, D, E, F, G, H, I, J
$$

The user preference list was as follows:

$$
1 C, 2 G, 3 E, 4 A, 5 J
$$

The recommender system recommended as follows:

$$
C, G, E, D, A
$$

In this study the performance of the system was given as follows per given service for each user:

\begin{tabular}{|l|l|l|}
\multicolumn{3}{|c|}{ TABLE I: RECALL AND PRECISION RESULTS FROM ONE USER } \\
\hline $\mathrm{k}$ & Recall @ $\mathrm{k}$ & Precision @ $\mathrm{k}$ \\
\hline 1 & $20 \%$ & $100 \%$ \\
\hline 2 & $40 \%$ & $100 \%$ \\
\hline 3 & $60 \%$ & $100 \%$ \\
\hline 4 & $60 \%$ & $75 \%$ \\
\hline 5 & $80 \%$ & $80 \%$ \\
\hline
\end{tabular}

The results from every user was added at every level $\mathrm{k}$ and averaged. The averages from each user were summed up and mean was obtained. The final results are shown in Table II.

TABLE II: MeAn AVerage of ReCAll and Precision Results From ALL USERS

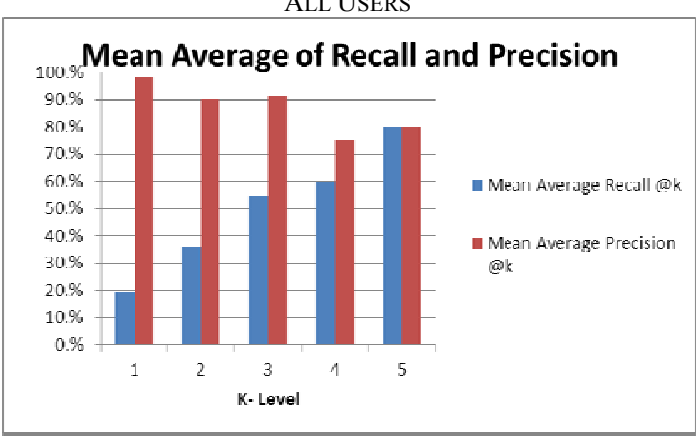

\section{CONCLUSION}

The research proposed a generic service location discovery recommender system that is used by mobile users especially in places that the user is not familiar with. The application was able to match the places where the user is able to get service with the user's taste. The system is able to adapt to the user's changing requirements. The results of the experiments conducted showed an impressive performance of the system.
In this generic service location recommender system for mobile users the accuracy of the recommendation is the most critical element of the system as much as the efficiency of the system must be acceptable. In future the system must be experimented with large number of users and service locations.

\section{REFERENCES}

[1] T. Bogers and A. V. D. Bosch, "Collaborative and content-based filtering for item recommendation on social bookmarking websites," in Proc. ACM RecSys '09 Workshop on Recommender Systems and the Social Web, New York, USA, 2009, pp. 9-16.

[2] A. Gunawardana and C. Meek, "A unified approach to building hybrid recommender systems," in Proc. the 2009 ACM Conference on Recommender Systems, New York, 2009, pp. 117-124.

[3] C. L. Huang and W. L. Huang, "Handling sequential pattern decay:Developing a two-stage collaborative recommender system," Electronic Commerce Research and Applications, vol. 8, pp. 117-129, 2009.

[4] T. Zuva, O. O. Olugbara, S. O. Ojo, and S. M. Ngwira, "Image content in location-based shopping recommender systems for mobile users," Advanced Computing: An International Journal (ACIJ), vol. 3, pp. 1-8, 2012.

[5] T. Zuva, S. O. Ojo, Seleman M. Ngwira, and K. Zuva, "A survey of recommender systems techniques, challenges and evaluation metrics," International Journal of Emerging Technology and Advanced Engineering, vol. 2, pp. 382-386, 2012.

[6] P. Melville and V. Sindhwani, "Recommender systems," in Encyclopedia of Machine Learning, S. Verlag, Ed., Berlin: Springer, 2010, pp. 1-9.

[7] M. J. Pazzani and D. Billsus, "Content-based recommendation systems," The Adaptive Web, Methods and Strategies of Web Personalization, pp. 325-341, 2007.

[8] J. B. Adair and M. Turnbull, "A Procedure for Calculating Great Circle Distances between Geographic Locations," Council for Advanced transportation Studies, the University of Texas at Austin, 1974.

[9] C. D. Manning, P. Raghavan, and H. Schutze, Introduction to Information Retrieval, Cambridge University Press, 2008.

[10] T. Mandl, "Recent developments in the evaluation of information retrieval system: moving towards diversity and practical relevance," Informatica, vol. 32, pp. 27-38, 2008.

[11] K. Zuva and T. Zuva, "Evaluation of information retrieval systems," International Journal of Computer Science \& Information Technology (IJCSIT), vol. 4, pp. 35-43, 2012.

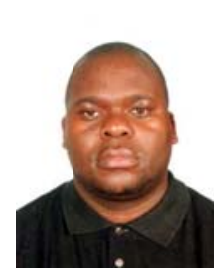

Tranos Zuva is with the Department of Computer Systems Engineering at Tshwane University of Technology, Soshanguve South Campus, and South Africa. He has published many articles in local and international conferences and journals.

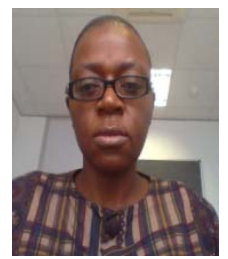

Keneilwe Zuva is working as a lecturer in the Department of Computer Science at the University of Botswana, Gaborone, Botswana. She has published many articles in local and international conferences and journals. 